\title{
Affinity Purification of RNA: Sequence-Specific Capture by Nonionic Morpholino Probes
}

\author{
J.M. Wages, Jr., G.M. Wages, P. Matthews, D. Weller and J. Summerton \\ AntiVirals, Corvallis, OR, USA
}

BioTechniques 23:1116-1121 (December 1997)

\begin{abstract}
Nucleic acid isolation for amplification-based diagnostics requires techniques that do not co-purify inhibitors of DNA polymerases. Also, other requirements for an ideal sample preparation technology include ease of use, capability for automation, high recovery and the use of nontoxic reagents. Affinity purification techniques provide high purification factor with minimal sample processing. Hybridization is the affinity interaction specific to nucleic acids and thus provides a uniquely advantageous method for purifying DNA or RNA for subsequent manipulation. Nonionic (morpholino) probes (Neu-Probes ${ }^{\mathrm{TM}}$; AntiVirals, Corvallis, OR, USA) have several unique hybridization properties, including resistance to $\mathrm{nu}$ cleases and the ability to hybridize independently of salt concentration. Therefore, such probes provide advantages over DNA probes for sample preparation by hybridization capture.

Three formats for hybridization-based purification of human immunodeficiency virus (HIV) RNA were evaluated using RNA transcripts spiked into crude lysates of normal human plasma. Indirect capture used streptavidin-coated microparticles to capture hybrids of biotinylated capture probes and HIV RNA. Direct capture used particles precoated with probes. In addition, a novel method for acceleration of sequence-specific hybridization was developed and shown to give consistently high recoveries.
\end{abstract}

\section{INTRODUCTION}

If DNA diagnostics are to become as widely used and as reliable as immunoassays, new techniques for nucleic acid isolation must be developed. This requirement is particularly acute for amplification-based diagnostics because of the pres- ence in clinical specimens of inhibitors of DNA polymerases. Heparin (1) and heme $(10,13)$ are frequently encountered in clinical extracts. Glycoproteins (24) and high concentrations of albumin (20) can be inhibitory. Nonhomologous DNA can also reduce the efficiency of polymerase chain reaction (PCR). This sort of inhibition is typically reversible through the use of hot-start protocols (5). Efficiency of PCR is monitored through the use of internal standards but at higher assay cost. A better approach to the inhibitor problem is to avoid copurifying them with the DNA or RNA to be amplified.

Nucleic acids can be extracted and amplified from a crude lysate (9). More commonly, they are purified by such techniques as organic extraction (e.g., acid guanidinium phenol; Reference 4), silica binding (2), salt precipitation (14) or cationic detergents (6). Total mRNA is typically purified by capture of all poly(A) sequences on solid phases containing poly(dT) (11). Immunoaffinity reagents have been used to capture intact viral particles prior to conventional extraction (3). A few reports have described the purification of specific mRNAs or viral nucleic acids by probe capture $(15,19)$, demonstrating the feasibility of using hybridization as an affinity purification technique for nucleic acids.

Recently, nonionic polymers for sequence-specific binding to nucleic acids have been described (peptide nucleic acids [PNA; References 16 and 17] and morpholino oligonucleotides [Neu-Probes ${ }^{\mathrm{TM}}$; AntiVirals, Corvallis, OR, USA; References 12 and 18]). Such molecules have unique properties as hybridization probes, including the ability to hybridize independently of ionic strength and essentially complete resistance to nucleases. We compare three formats for RNA purification by hybridization-based capture using uncharged morpholino probes. 


\section{MATERIALS AND METHODS}

\section{RNA Transcripts}

RNA transcripts were prepared using the MEGAscript ${ }^{\mathrm{TM}}$ T7 Kit (Ambion, Austin, TX, USA) following the manufacturer's recommended procedure. Initial experiments used a 550-nucleotide (nt) transcript from human immunodeficiency virus (HIV) gag (pBENN7; Reference 7), and experiments with multiple specific-capture probes used a 750-nt RNA. Also, a nonhomologous RNA of similar size was prepared that contained sequences of rabbit $\alpha$-globin. Unincorporated nucleotides were removed using two passes through ProbeQuant $^{\mathrm{TM}}$ G-50 Micro Columns (Pharmacia Biotech, Piscataway, NJ, USA), followed by lithium chloride precipitation. Poly(A) polymerase (Life Technologies, Gaithersburg, MD, USA) was used to add a poly(A) tail. Integrity of the transcripts and approximate tail lengths (at least $100 \mathrm{nt}$, by comparison with RNA standards) were monitored by electrophoresis on $4 \%$ polyacrylamide/7 M urea gels.

Lysates of normal human plasma were prepared with $3 \mathrm{vol}$ of PUREscript ${ }^{\circledR}$ Cell Lysis Solution (Gentra Systems, Minneapolis, MN, USA), which were first titrated to $\mathrm{pH} 7.5$ with $10 \mathrm{~N} \mathrm{NaOH}$. RNA $\left(10^{4}\right.$ counts per minute [cpm]; alternatively, $10^{4}$ copies in experiments using a PCR endpoint) was then added to the plasma lysate ( $50 \mu \mathrm{L}$ plasma $+150 \mu \mathrm{L}$ lysis solution). All experiments (except that for which results are described in the legend to Figure 1) used an RNA-spiked plasma lysate. For capture by any of the formats described in this report, $100 \mu \mathrm{g}$ of streptavidin-coated 2.8- $\mu \mathrm{m} \mathrm{M-280} \mathrm{Super-}$ paramagnetic Dynabeads ${ }^{\circledR}$ (Dynal, Lake Success, NY, USA) and incubation at $37^{\circ} \mathrm{C}$ with constant shaking for $30 \mathrm{~min}$ were used. Capture particles were magnetically separated using a magnetic particle concentrator (MPC ${ }^{\circledR}$-E; Dynal) and washed

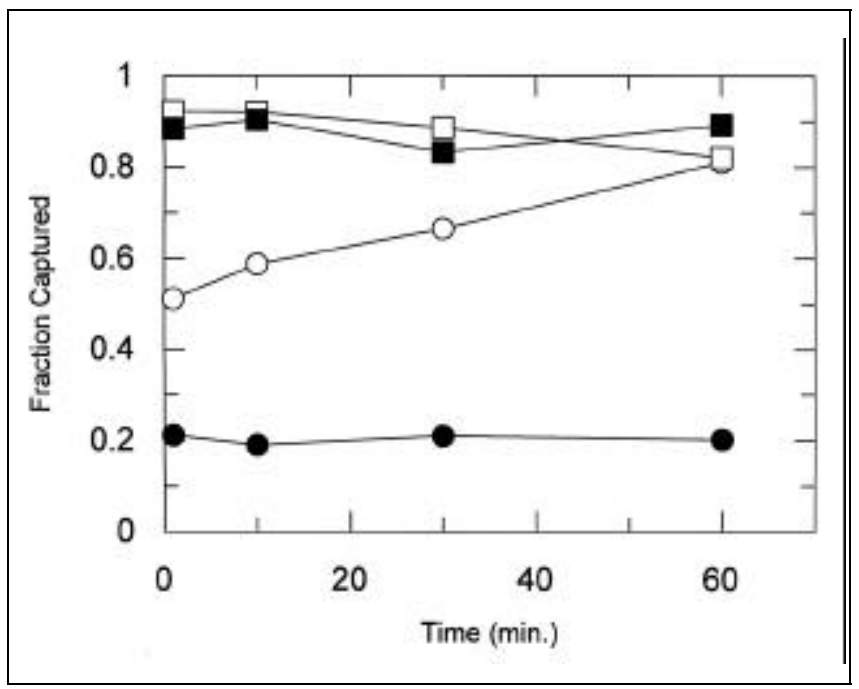

Figure 1. Capture of poly(A) RNA at high and low ionic strength. Capture was compared in high-salt $(0.5 \mathrm{M} \mathrm{LiCl}, 0.5 \%$ lithium dodecyl sulfate, $100 \mathrm{mM}$ Tris-HCl, pH 8.0, 10 mM EDTA) and low-salt (1 mM EDTA, pH 8.0) solutions. Radiolabeled RNA was diluted to ca. $10^{4} \mathrm{cpm} / 10 \mu \mathrm{L}$, corresponding to approximately $0.5-5 \mathrm{nM}$. Beads were washed three times in 1 mM EDTA ( $\mathrm{pH} 8.0$ ) to remove storage solution, and $50 \mu \mathrm{g}$ were added to the sample. Following incubation at $37^{\circ} \mathrm{C}$ with constant shaking, beads were magnetically separated. The supernatant was counted, and the fraction captured was calculated. Efficient capture was obtained within $1 \mathrm{~min}$ in high-salt buffer. Morpholino $\mathrm{U}_{25}$, in contrast to $\mathrm{dT}_{25}$, was also able to hybridize in 1 mM EDTA. 
three times with RNase-free water. For particles containing $\mathrm{U}_{25}$ and a cleavable linker, beads were washed with $50 \mathrm{mM}$ dithiothreitol (DTT).

\section{Capture Probes}

HIV sequence-specific probes (antisense) were designed to bind upstream of the SK 462 primer sequence (23), thus outside the region targeted for amplification. Morpholino capture probes were synthesized with $5^{\prime}$ biotin and a long spacer (polyethylene glycol [PEG]-3400; Shearwater Polymers, Huntsville, AL, USA) between biotin and the probe sequence. The quality of each oligonucleotide following HPLC purification was assessed by matrix-assisted laser desorption ionization mass spectroscopy with time of flight analyzer (MALDI-TOF). Stock solutions (1 mM) in RNase-free water were stored at $4{ }^{\circ} \mathrm{C}$. The probe sequences were as follows (HIV sequence positions refer to the HXB2 isolate, whose sequence was obtained from the Los Alamos HIV sequence database): ${ }_{m o}(\mathrm{U})_{25}: 5^{\prime}$-biotin-S-S-PEG- ${ }_{\text {mo }}(\mathrm{U})_{25}$; HIV-CP1:

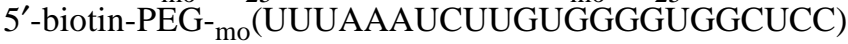
(nt 1342-1320); HIV-CP2: 5'-biotin-PEG-mo (GAAAACAUGGGUAUCACUUCUGGGC) (nt 1306-1282); HIV-CP3: 5'biotin-PEG- ${ }_{\text {mo }}$ (UGCGAAUCGUUCUAGCUCCCUG) (nt 923-902).

\section{Capture Formats and Particles}

Three hybridization capture formats were evaluated. For indirect capture, $10 \mu \mathrm{L}$ of RNA (approximately $0.5-5.0 \mathrm{nM}$, $10^{4} \mathrm{cpm}$ ) were mixed with $10 \mu \mathrm{L}$ of $30 \mathrm{nM}$ probe solution. Hybridization was allowed to occur in solution for $30 \mathrm{~min}$ at $37^{\circ} \mathrm{C}$, at which time, $10 \mu \mathrm{L}$ of $10-\mu \mathrm{g} / \mu \mathrm{L} \mathrm{M}-280$ Dynabeads were added. Capture occurred during a 30-min incubation at

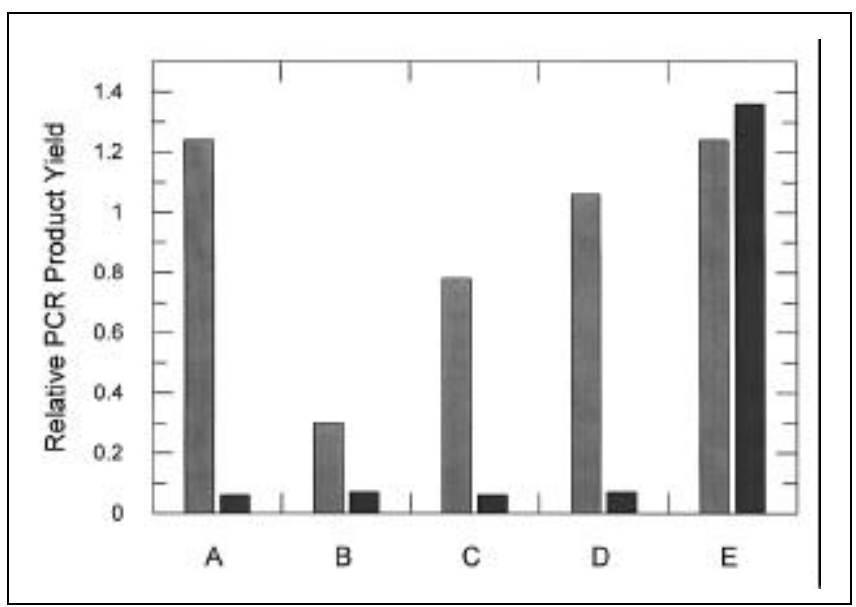

Figure 2. Hybridization capture avoids co-purification of PCR inhibitors. Plasma was spiked with high levels of heme $(100 \mu \mathrm{g} / 50 \mu \mathrm{L}$ plasma; Sigma Chemical, St. Louis, MO, USA) and heparin $(20 \mu \mathrm{g} / 50 \mu \mathrm{L}$ plasma; Sigma Chemical) and was then extracted using: (A) acid guanidinium phenol (Tri Reagent ${ }^{\circledR}$ LS; Molecular Research Center, Cincinnati, OH, USA); (B) salt precipitation (PUREscript); (C) silica binding (HCV RNA Isolation Kit; Qiagen, Chatsworth, CA, USA); (D) cationic detergent (Catrimox ${ }^{\mathrm{TM}_{-}}$14; Iowa Biotechnology, Iowa City, IA, USA) using formamide extraction from the pelleted RNA (6); and (E) hybridization-based capture on $\mathrm{U}_{25}$ particles. Extracts were spiked with poly(A) HIV RNA corresponding to $10^{4}$ copies per reaction and assessed for inhibition by RT-PCR using $50 \mu \mathrm{L}$ plasma equivalent per reaction. The plotted values are relative fluorescence units from the capture assay as a fraction of control reactions ( $10^{4}$ copies). Only hybridization-based purification avoids co-purifying both heme and heparin. room temperature with constant shaking.

Direct capture used particles previously saturated with probes, following the manufacturer's suggested protocol for coating the particles, but using $0.6 \mathrm{pmol}$ probe $/ \mu \mathrm{g}$ particles to ensure maximal coating. No direct assessment of probe loading was made, and the possibility exists that binding capacity of M-280 for DNA oligonucleotides is different from uncharged morpholino oligonucleotides of similar lengths. Higher binding capacities for biotinylated morpholino oligonucleotides, for example, would result in particles showing a higher efficiency of capture of complementary sequences.

A third capture format, which we designated the multipleprobe capture system, exploits the rapid kinetics of capture by poly(U) to give an acceleration of hybridization to sequencespecific probes bound to the same particles. Polyadenylated RNA bound only through the poly(U) sequence is released through a cleavable disulfide linker using DTT, leaving only specifically hybridized RNA bound to the particle. Particles were coated with $\mathrm{U}_{25}$ and either HIV-CP1 or an equimolar mixture of HIV-CP1, -CP2 and -CP3. M-280 particles bearing no capture probes were found to not capture appreciable amounts of HIV RNA under these conditions (data not shown). Using radiolabeled, nonhomologous RNA, beads carrying only HIV-specific probes were found to capture $\leq 2 \%$ of applied cpm (data not shown).

\section{PCR Methods}

For assessment of capture of RNA at low copy number, reverse transcription (RT)-PCR was performed by standard methods using 30 cycles and $0.2 \mu \mathrm{M}$ each of primers SK 462 and 5'-biotin SK 431 (Reference 23; BioSource, Camarillo, CA, USA). For PCR product analysis, an affinity-based hybrid capture assay (22) was used with fluorescence detection of alkaline phosphatase using 4-methylumbelliferyl phosphate. Typically, 100 copies of RNA could be detected by these methods, and 100-104 copies covered the exponential phase of PCR.

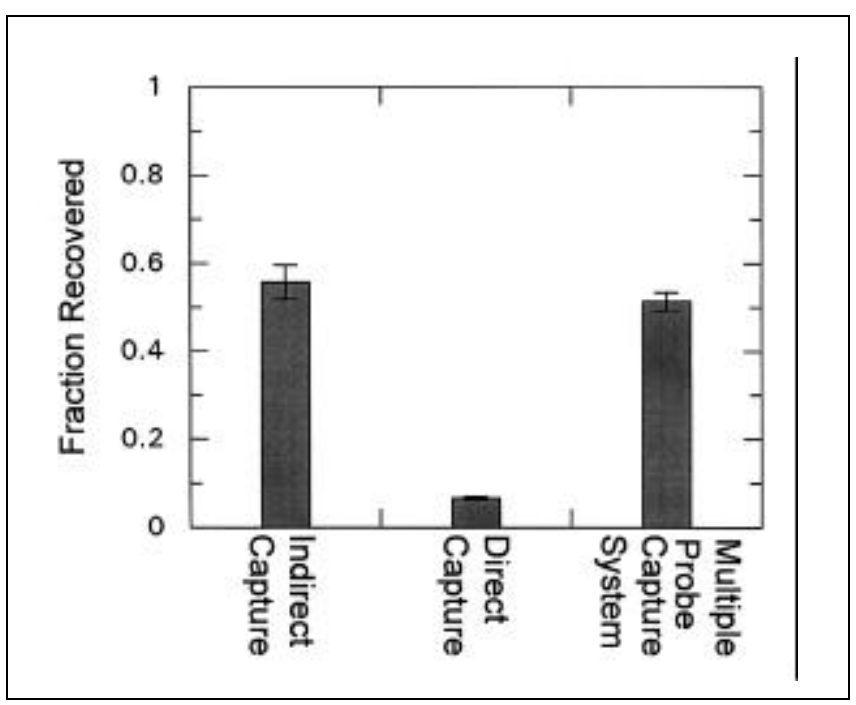

Figure 3. Comparison of three hybridization capture formats. Poly(A) HIV RNA $\left(10^{4} \mathrm{cpm}\right)$ was spiked into plasma lysates in triplicate and purified using each of the three capture formats. Multiple HIV-specific probes (HIV$\mathrm{CP} 1,-\mathrm{CP} 2$ and -CP3) were used in each case. Recoveries were normalized to the percentage of cpm accounted for by the major band in polyacrylamide gel electrophoresis of the RNA transcripts (85.4\%). 


\section{RESULTS}

\section{Capture in Low-Salt Solution}

The efficiencies of capture by $\mathrm{dT}_{25}$ (Dynal) and morpholino $\mathrm{U}_{25}$ were compared in high-salt and low-salt hybridization buffers. Figure 1 indicates that efficient capture was obtained within 1 min in high-salt buffer, using either DNA or morpholino capture oligonucleotides. Morpholino $\mathrm{U}_{25}$, in contrast to $\mathrm{dT}_{25}$, was also able to hybridize at low ionic strength. Since experiments (Wages, unpublished) show that morpholino probes hybridize to DNA targets with kinetics that are not dependent on salt concentration, we attribute this somewhat slower binding at very low ionic strength to an effect on the particles rather than the probes. Successful hybridization in 1 mM EDTA demonstrates the potential for hybridization at low ionic strength where nucleic acid secondary structures are expected to be destabilized.

\section{Co-Purification of Inhibitors}

To assess co-purification of inhibitors by various RNA purification techniques, plasma was spiked with high levels of heme or heparin. As shown in Figure 2, only hybridizationbased purification avoids co-purifying these inhibitors.

\section{Multiple-Probe Capture System}

Initial experiments (21) with a single specific-capture probe and $U_{25}$ gave selective retention of HIV RNA but low recovery (37\%). In separate experiments with RNA bound to particles having only HIV-CP1, some loss was observed with heating at $37^{\circ} \mathrm{C}$. This suggested that higher recoveries could be obtained using a longer capture probe, a probe with higher $\mathrm{T}_{\mathrm{m}}$ or multiple-capture probes. Particles were subsequently prepared by coupling probes HIV-CP1, HIV-CP2, HIV-CP3 and $\mathrm{U}_{25}$ to M-280 Dynabeads. Increased recovery was obtained $(51.4 \%)$, suggesting that multiple-capture probes facilitate retention of RNA on the particles.

\section{Comparison of Three Capture Formats}

Capture and recovery of poly(A) HIV RNA were then compared for the three capture formats. Figure 3 shows that capture and recovery were higher with indirect capture $(56 \%)$ or the multiple-probe capture system $(51 \%)$ than with direct capture $(6.6 \%)$. The presence of multiple specific probes for direct capture resulted in only marginal improvements in capture and recovery. Although multiple probes increased the recovery of direct capture from $0.8 \%$ (essentially background) to $6.6 \%$, under no conditions tested did direct capture on M280 particles approach the efficiency attained with either of the other methods.

\section{DISCUSSION}

We present methods for hybridization-based sample preparation for RT-PCR and demonstrate that such techniques 
avoid co-purification of PCR inhibitors. This suggests that clinical assays will benefit from hybridization-based sample preparation, which will reduce the frequency of false negatives resulting from co-purification of heme or polyanionic polysaccharides. Such techniques may also be useful for the analysis of archival specimens collected using heparin anticoagulant and for the purification of DNA from environmental samples.

The multiple-probe capture system, which uses a rapidly hybridizing, generic probe to accelerate hybridization to sequence-specific probes, was developed as a technique for selective, high-recovery purification of specific polyadenylated sequences. Such a technique might have applications for selecting specific mRNAs from total RNA. Other hybridization accelerants can be envisioned that would use affinity interactions such as dye binding (8) or cationic capture to bring RNA from solution into proximity to specific probes. As long as the affinity reagents do not interfere with hybridization, such approaches appear feasible.

These capture formats should support the use of DNA probes and the novel morpholino oligonucleotides described in this report. Unlike DNA probes, the pairing of uncharged probes to their target sequences is relatively independent of salt concentration. This makes possible capture of targets under conditions that destabilize RNA secondary structure and thereby expose otherwise unavailable target sequences. This property is likely to be advantageous for target RNAs with stable secondary structures, such as rRNA or the untranslated regions of mRNAs. In the case of viruses, such as hepatitis C virus $(\mathrm{HCV})$, the untranslated regions contain some of the most conserved sequences, which are desirable diagnostic targets. Degenerate capture probes may also prove useful for gene or new pathogen discovery research, using probes based on known sequence motifs. Since morpholino oligonucleotides are not extended by polymerases, even high concentrations of unrelated morpholino sequences do not interfere with subsequent PCR.

\section{ACKNOWLEDGMENTS}

We wish to thank Dr. Elisabeth Barofsky (Department of Agricultural Chemistry, Oregon State University) for performing the mass spectroscopy of morpholino probes and Cynthia Herzog for help in preparation of the manuscript and figures. In addition, we wish to thank the entire Chemistry group at AntiVirals, Inc.: Scott Davis, Cheryl Dawley, Dave Dawley, Christina Fox, Mary Kay Gleicher, Makarand Gore, Sung-Ben Huang, Jordana Jacobs, Cheri Kiesecker, Elizabeth Lai, Joe Maxwell, Avinash Phadke, Linda Uhlir and Doreen Weller.

\section{REFERENCES}

1.Beutler, E., T. Gelbart and W. Kuhl. 1990. Interference of heparin with the polymerase chain reaction. BioTechniques 9:166.

2.Boom, R., C.J.A. Sol, M.M.M. Salimans, C.L. Jansen, P.M.E. Wertheim-van Dillen and J. van der Noordaa. 1990. Rapid and simple method for purification of nucleic acids. J. Clin. Microbiol. 28:495-503.

3.Brown, V.K. and B.H. Robertson. 1990. Immunoselection of clinical specimens containing virus followed by polymerase chain reaction amplification and rapid direct sequencing. BioTechniques 8:262-264.

4.Chomczynski, P. and N. Sacchi. 1987. Single-step method of RNA isolation by acid guanidinium thiocyanate-phenol-chloroform extraction. Anal. Biochem. 162:156-159.
5.Chou, Q., M. Russell, D.E. Birch, J. Raymond and W. Bloch. 1992. Prevention of pre-PCR mis-priming and primer dimerization improves low-copy-number amplifications. Nucleic Acids Res. 20:1717-1723.

6.Dahle, C.E. and D.E. Macfarlane. 1993. Isolation of RNA from cells in culture using Catrimox-14TM cationic surfactant. BioTechniques 15:11021105

7.Gendelman, H.E., W. Phelps, L. Feigenbaum, J.M. Ostrove, A. Adachi, P.M. Howley, G. Khoury, H.S. Ginsberg and M.A. Martin. 1986. Trans-activation of the human immunodeficiency virus long terminal repeat sequence by DNA viruses. Proc. Natl. Acad. Sci. USA 83:9759-9763.

8.Harding, J.D., G. Gebeyehu, R. Bebee, D. Simms and L. Klevan. 1989. Rapid isolation of DNA from complex biological samples using a novel capture reagent-methidium-spermine-sepharose. Nucleic Acids Res. 17:6947-6958.

9.Higuchi, R. 1989. Rapid, efficient DNA extraction for PCR from cells or blood. Amplifications 2:1-3.

10.Higuchi, R. 1989. Simple and rapid preparation of samples for PCR, p. 31-38. In H.E. Erlich (Ed.), PCR Technology: Principles and Applications for DNA Amplification. Stockton Press, New York.

11.Hornes, E. and L. Korsnes. 1990. Magnetic DNA hybridization properties of oligonucleotide probes attached to superparamagnetic beads and their use in the isolation of poly(A) mRNA from eukaryotic cells. Genet. Anal. Tech. Appl. 7:145-150.

12.Hudziak, R.M., E. Barofsky, D.F. Barofsky, D.L. Weller, S.-B. Huang and D.D. Weller. 1996. Resistance of morpholino phosphorodiamidate oligomers to enzymatic degradation. Antisense Nucleic Acid Drug Dev. 6:267-272.

13.Lin, L., Y. Gong, K. Metchette, G.D. Cimino, J.E. Hearst and S.T. Isaacs. 1993. Simple and rapid sample preparation methods for whole blood and blood plasma, p. 505-616. In D.H. Persing, T.F. Smith, F.C. Tenover and T.J. White (Eds.), Diagnostic Molecular Biology. Academic Press, New York.

14.Linnen, J., J. Wages, Jr., Z-Y. Zhang-Keck, K.E. Fry, K.Z. Krawczynski, H. Alter, E. Koonin, M. Gallagher et al. 1996. Molecular cloning and disease association of hepatitis $\mathrm{G}$ virus: a transfusion-transmissible agent. Science 271:505-508.

15.Muir, P., F. Nicholson, M. Jhetam, S. Neogi and J.E. Banatvala. 1993. Rapid diagnosis of enterovirus infection by magnetic bead extraction and polymerase chain reaction detection of enterovirus RNA in clinical specimens. J. Clin. Microbiol. 31:31-38.

16.Nielsen, P.E., M. Egholm, R.H. Berg and O. Buchardt. 1991. Sequence-selective recognition of DNA by strand displacement with a thymine-substituted polyamide. Science 254:1497-1500.

17. Ørum, H., P.E. Nielsen, M. Jørgensen, C. Larsson, C. Stanley and T. Koch. 1995. Sequence-specific purification of nucleic acids by PNA-controlled hybrid selection. BioTechniques 19:472-480.

18.Summerton, J.E. and D.D. Weller, Inventors. Uncharged MorpholinoBased Polymers Having Phosphorus Containing Chiral Intersubunit Linkages. U.S. Patent No. 5185444. 1993 February 9.

19.Thompson, J.D., S. Decker, D. Haines, R.S. Collins, M. Feild and D. Gillespie. 1989. Enzymatic amplification of RNA purified from crude cell lysate by reversible target capture. Clin. Chem. 35:1878-1881.

20. Vandenvelde, C., R. Scheen, M. Defoor, M. Duys, J. Dumon and D. Van Beers. 1993. Suppression of the inhibitory effect of denatured albumin on the polymerase chain reaction by sodium octanoate: application to routine clinical detection of hepatitis B virus at its infectivity threshold in serum. J. Virol. Methods 42:251-264.

21.Wages, J., G.M. Wages, D. Weller and J. Summerton. 1995. Sample preparation for nucleic acid diagnostics: sequence-specific capture by non-ionic morpholino oligos. Clin. Chem. 41:1683.

22.Wages, J.M., L. Dolenga and A.K. Fowler. 1993. Electrochemiluminescent detection and quantitation of PCR-amplified DNA. Amplifications 10:1-6.

23.Whetsell, A.J., J.B. Drew, G. Milman, R. Hoff, E.A. Dragon, K. Adler, J. Hui, P. Otto et al. 1992. Comparison of three nonradioisotopic polymerase chain reaction-based methods for detection of human immunodeficiency virus type 1. J. Clin. Microbiol. 30:845-853.

24.Woodard, D., A. Walters, D. McLaurin, T. Walker, C. Dean and D. Malinowski. 1995. Inhibitory effect of glycoproteins on strand displacement amplification. Clin. Chem. 41:1680.

Address correspondence to Paula I. Matthews, AntiVirals, Inc., 4575 SW Research Way, Suite 200, Corvallis, OR 97333, USA.Internet: matthep@antivirals.com 